\title{
Structure Design for Modular Platform and Capability on the USUsat 2 Micro-satellite
}

\author{
Joël Quincieu \\ USUsat 2 Chief Engineer \\ Space Dynamics Laboratory, Utah State University, Logan, Utah \\ joel.quincieu@sdl.usu.edu
}

Academic Advisors: Dr. Charles Swenson (PI), Dr. Rees Fullmer (co-PI), Dr. Todd Mosher (co-PI)

Department of Mechanical and Aerospace Engineering

Utah State University, Logan, UT 84322-1600

\begin{abstract}
In the last fifteen years, small satellites have opened a window through which the aerospace industry can rapidly access low earth orbit at a fraction of the cost required by large spacecrafts. Due to its extensive experience with the Space Dynamics Laboratory (SDL), Utah State University (USU) has given students the opportunity to experience the design of such small satellites, nanosatellites or micro-satellites in technical terms. Through the University Nanosat programs organized mainly by the Air Force Research Laboratory (AFRL), USU students have now been involved in two satellite designs since 1999. The author has been and is contributing to both programs.

The current program, USUsat 2, has been partially derived from the experience of the previous USUsat I/ION-F program. This paper deals with the USUsat 2 design and more specifically on the manufacturing side and the structure for a modular platform. The author, who has been working as the chief engineer for the program, proposes an approach with a modular design with new structure. The design is intended by the author to have the capability of becoming a micro-satellite platform that future programs could reuse with a minimum amount of upgrade.
\end{abstract}

\section{Introduction}

The USUsat 2 design strategy is primarily focused on developing the capability to carry out a mission that initially is not completely defined. This method calls for selecting technology prior to mission definition. For this approach, the cost reduction can be achieved by defining what can be implemented in terms of capability or available technology. The reverse, more traditional, method would be to design the technology based on the mission, but this means that the satellite is directly dependant on the mission and the design, therefore, becomes variable. The design-to-capability approach achieves cost reduction by focusing on available, existing resources and that the mission must adapt to these resources. This approach works well for fixed budget and low cost programs. The concept can be advantageous for a low budget project such as a university program, but is also advantageous for future programs that can use the existing platform that is already designed and documented. Similar approaches have been implemented in the personal computer (PC) industry, where multiple vendors can build a PC or add additional hardware. Along the same lines, the USUsat 2 design is oriented toward a primary science (ionospheric) mission, but has the flexibility to include additional payloads. Since the prospect of receiving additional payloads - such as science or technological experiments - is highly possible during the design timeline, the USUsat 2 satellite must be able to adapt to provide the functional resources such as power, data handling, communication interface, and mechanical integrity that are required.

The design-to-capability concept can also apply to a satellite with a modular platform that is capable of adapting to missions by re-using existing modules and components. The author has proposed that the USUsat 2 design respond to such a platform concept. Creating a modular design carries a important 
advantages in reducing costs. Once the initial unit is built, the design is proven and documented, the cost of starts-up, infrastructure, manufacturing and documentation are then minimized for following units.

To provide a design platform that will reduce the cost, shorten lead-time, and shorten the design and manufacturing processes, two elements are essential:

- A micro-satellite bus design must be reusable

- The design must be based on a flexible architecture or configuration (packaging), that can integrate the bus and the payload, and that can be re-produced with a minimum amount of modification (modularity) and cost.

\section{The USUsat 2 micro-satellite mission} overview for the UNIII program

The USUsat 2 micro-satellite is in the process of being designed as a participant of the University Nanosate III competition (UNIII), a national competition. The mission selected by the USU team for this competition was influenced primarily by current research of Dr. Swenson to study the ionosphere. The same scientific mission was initially going to fly on a previous program, USUsat I / Ion-F. The USUsat 2 satellite is designed to be launched on NASA's Space Shuttle in 2006 to a $380 \mathrm{~km}$ orbit with a $53^{\circ}$ inclination.

\section{The basic sub-systems of the modular bus}

The basic sub-components that the nanosatellite requires to perform minimum tasks make up the bus, the core of the spacecraft. This core, the "spine" shown in Figure 3-1, represents all the necessary equipment or basic components to ensure the operation of the satellite once in space. The design of the spacecraft core does not need to be frozen at a certain time; rather, it must have the flexibility to be modified, improved, or evolved over time or for each new mission. For example, the ADCS for one mission could require a lower degree of accuracy than another, and therefore the design requirements could be relaxed. Once the bus is validated for flight use, it can be reused and adapted for other missions without "reinventing the wheel" and starting all over.

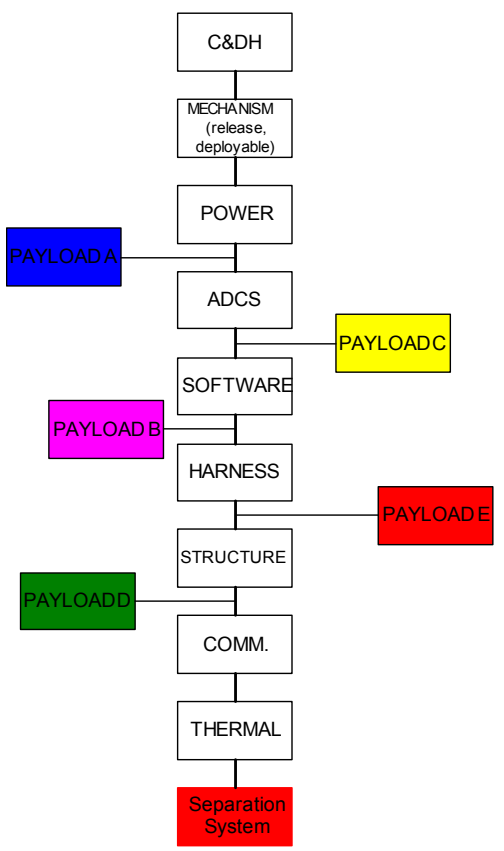

Figure 3-1. The bus or "spine" to support payloads and mission

A spacecraft bus, once validated on-orbit, can be adapted to new missions and additional payloads. Surrey University has successfully demonstrated this concept during a decade of activity. The reuse of the design reduces supporting hardware, tooling, analysis models (thermal, structural), documentation (test plans, assembly plans, drawings and schematics). This reuse will improve the design/manufacturing life cycle and provide rapid progress from conceptual design to detailed design.

\section{$4 \quad$ Modular assembly on USUsat 2}

In addition to traditional sub-systems requirements, the author proposed the USUsat 2 micro-satellite incorporate more of the modular platform concept in the structure than its predecessor. Incorporating the modular platform concept would enable an organization, such as USU, to produce one satellite per year. To do this, the USUsat 2 design revolves around core modules with components that can be pre-assembled and pre-tested. The modules are made of pre-mounted components on common structural panels. By maintaining common geometry, the modules could re-use the same tooling and test equipment. The modules can be rearranged, with their respective panel, with other modules within the baseline USUsat 2 model shown in Figure 4-1. 


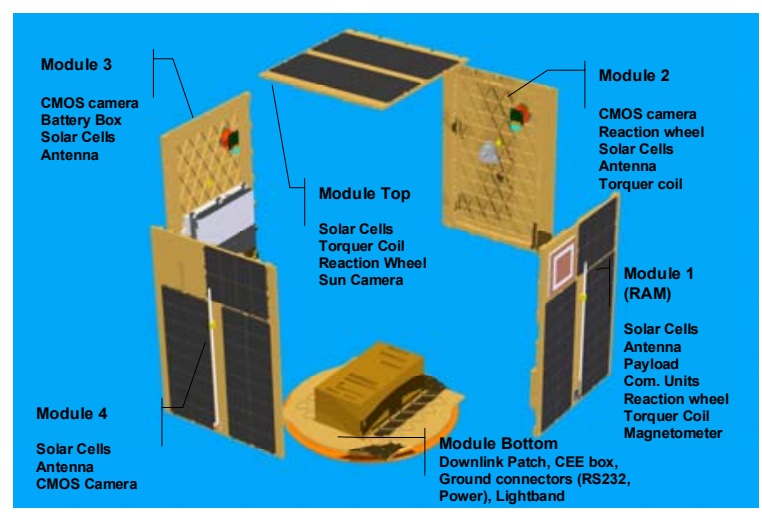

Figure 4-1. Modular design of the USUsat 2, total mass of $24 \mathrm{~kg}$

For the USUsat 2 design, the modules are pre-assembled and pre-wired, with only one connector for input and output (Intra-modules connector). Figure 4-2 shows a sample module layout. With this configuration, the modules can undergo full mechanical and functional testing prior to final integration. Modules are then considered as "limbs" of the spacecraft, with each "limb" a fully design and tested assembly.

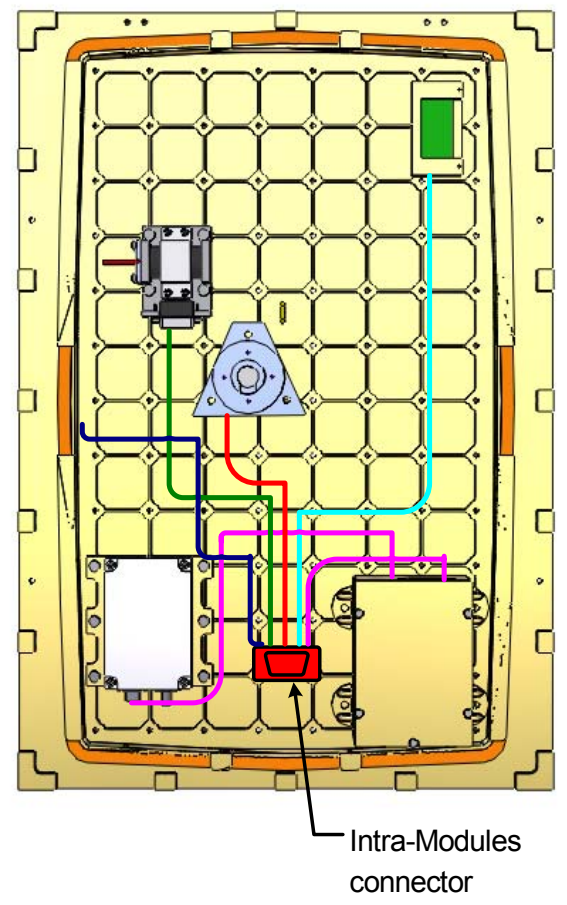

Figure 4-2. Harness routing using the standard bolt pattern on USUsat 2 thus avoiding the use of tape.
5 DFMA: Design for Manufacturing and Assembly

During the USUsat 2 design process, the author encouraged the team to apply a Design for Manufacturing and Assembly (DFMA) approach at all level of sub-assemblies and assemblies. The DFMA approach invites the team to apply concurrent engineering methods with a set of standards that will simplify the design and provide a more productive environment. The author has implemented standards in the design of the USUsat 2 micro-satellite to maintain a lean process. As an example, only three types of fasteners are used on the entire program. The assembly approach through modules on USUsat 2 is another example of this appraoch. The application of DFMA can be summarized by the following points.

- Design for a minimum number of parts.

- Develop a modular design.

- Minimize part variations.

- Design parts to be multi-functional.

- Design parts for multi-use.

- Design parts for ease of fabrication.

- Avoid separate fasteners.

- Minimize assembly directions; design for topdown assembly.

- Maximize compliance; design for ease of assembly.

- Evaluate assembly methods.

- Eliminate or simplify adjustments.

- Avoid flexible components.

\section{USUsat 2: The structure}

The primary structure of the USUsat 2 microsatellite, shown in Figure 6-1, follows the USUsat I model by using a machined, closed-grid pattern to provide a lightweight characteristic. The USUsat I structure was made of aluminum with a pattern of isometric triangles (IsoGrid) cut in order to reduce mass. The principal problem with the USUsat I structure is that specific locations for attaching components had to be added between the IsoGrid, adding to the complexity and requiring additional machining operation. This technique also forced the design to "freeze" early, at the CAD level and did not tolerate any error in misalignment when the structure was completed. There were also no specific locations or attachment holes present to hold a balance mass, in case the spacecraft was not in equilibrium. The 
harness was designed to be held with adhesive, which did not provide an easy configuration for disassembly, if required. Adhesives are also not the most desirable due to outgassing. The USUsat I design primarily addressed mass and structural concerns but did not address integration, versatility and modular purposes. When, for example, an additional instrument was manifested on the ION/F program after the structure was designed and built, one of the panels had to be redesigned to provide attachment location for the specific hardware.

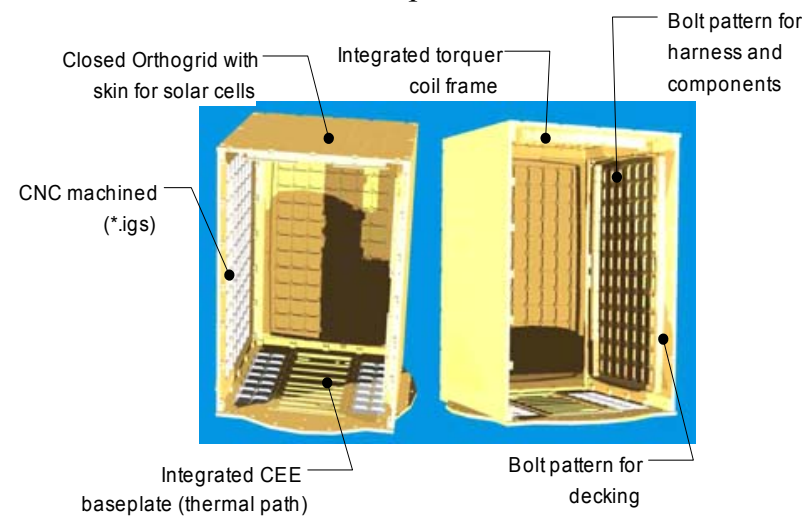

Figure 6-1. USUsat 2 structure features, $\sim 9 \mathrm{~kg}$, AL 6061-T6

For USUsat 2, the author proposed a more modular approach for the primary structure (see Figure 6-1). First, in order to give to the design team more versatility in locating internal components, a standard threaded bolt pattern (8-32") was placed within the grid, using fixed dimensions that are standard across the entire spacecraft. This provides the freedom to vary the position of components even after the structure is built. Masses used to balance the spacecraft can now be freely located without disturbing the current packaging. The bolt pattern has been changed by the author from an IsoGrid to a closed OrthoGrid configuration (see Figure 6-2, 3) which used square shapes instead of triangle shapes.

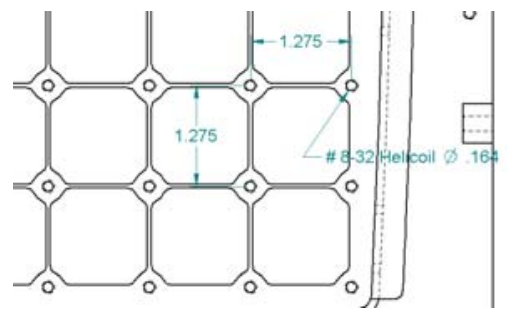

Figure 6-2. Standard bolt pattern between OrthoGrid on the structure
The OrthoGrid pattern allows internal units to be attached at four points. Using a four point mounting interface provides additional safety; if a bolt fails on a unit, three other points are able to secure it. As the bolt pattern is perfectly isometric, components can now be rotated in place, and can be adjusted as desired.

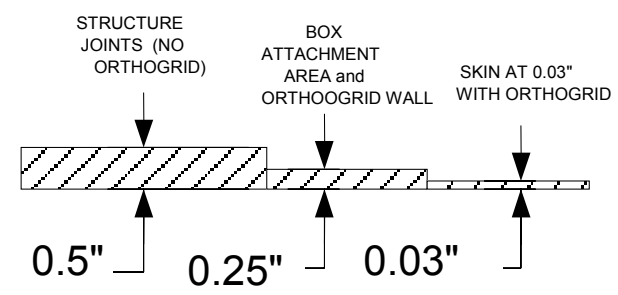

Figure 6-3. USUsat 2 structure thicknesses.

The previous IsoGrid configuration required the design engineers to follow the triangular pattern when designing internal units, which in most instances are non-symmetric with square or rectangular components geometry.

The author also recommended that the bolt pattern penetrate the panel so that external components could be attached as well. The bolt pattern can be used not only to attach components, but it also provides access from the inside to the outside for solar cell wiring. As the harness location is difficult to define in the early stage of the design, the bolt pattern now gives additional options to where the wiring needs to be attached and routed. As the bolt pattern is tapped (with Helicoil for reinforcement) with one set of thread size, the subsystem engineers will only have one option for what fasteners to use, thus eliminating the process and time spent of selecting fasteners optimized for a specific component. Thus, the standard is set and all engineers must adhere it when attaching any component on the spacecraft. Only three sizes of thread were used in the USUsat 2 design. The structure is made with only two thread sizes: $10-32$, 8-32. A 4-40 size is used in designing at the components level. Fasteners are vented to prevent the trapping of gasses that could cause problems during thermo-vacuum testing or depressurization during launch. With three standard sizes, the design engineers will not spend extra time searching for specific fasteners or their respective tooling. This aspect allows the design to becomes leaner and simpler. Only three Allen wrench sizes are needed to assemble the whole satellite. 


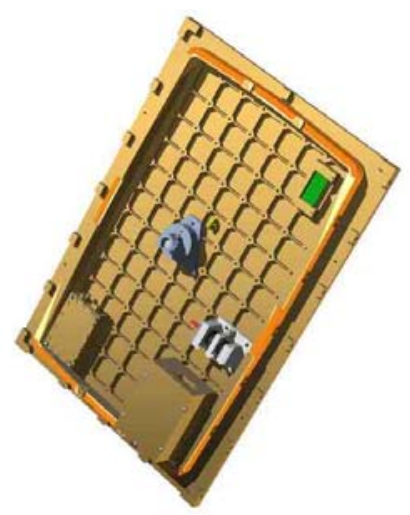

Figure 6-4. Module 1 with torquer-coil integrated in the structure. The coils (in orange color) are directly wound around the L-shape frame machined with the structure. Conventional zipstraps are used to attach the coil.

The assembled spacecraft structure is a

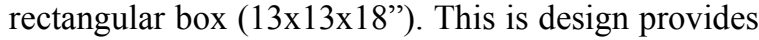
higher efficiency (compared to a hexagonal shape) for packaging since most of the internal units are rectangular. The bottom panel structure also integrates the base plate of the Common Electronic Enclosure (CEE, or flight computer) thus avoiding the adaptation of an external component and improving the thermal conductivity. This panel, with the integrated flight computer base plate, is shown in Figure 6-5.

The circular bolt pattern of the Lightband separation system is also directly integrated in the structure; again avoiding an external adaptor plate. The structure also has integrated coil frames on two side panels and the top panel. The wires for the drytorque coils are wrapped directly around the structure, as shown in Figure 6-4.

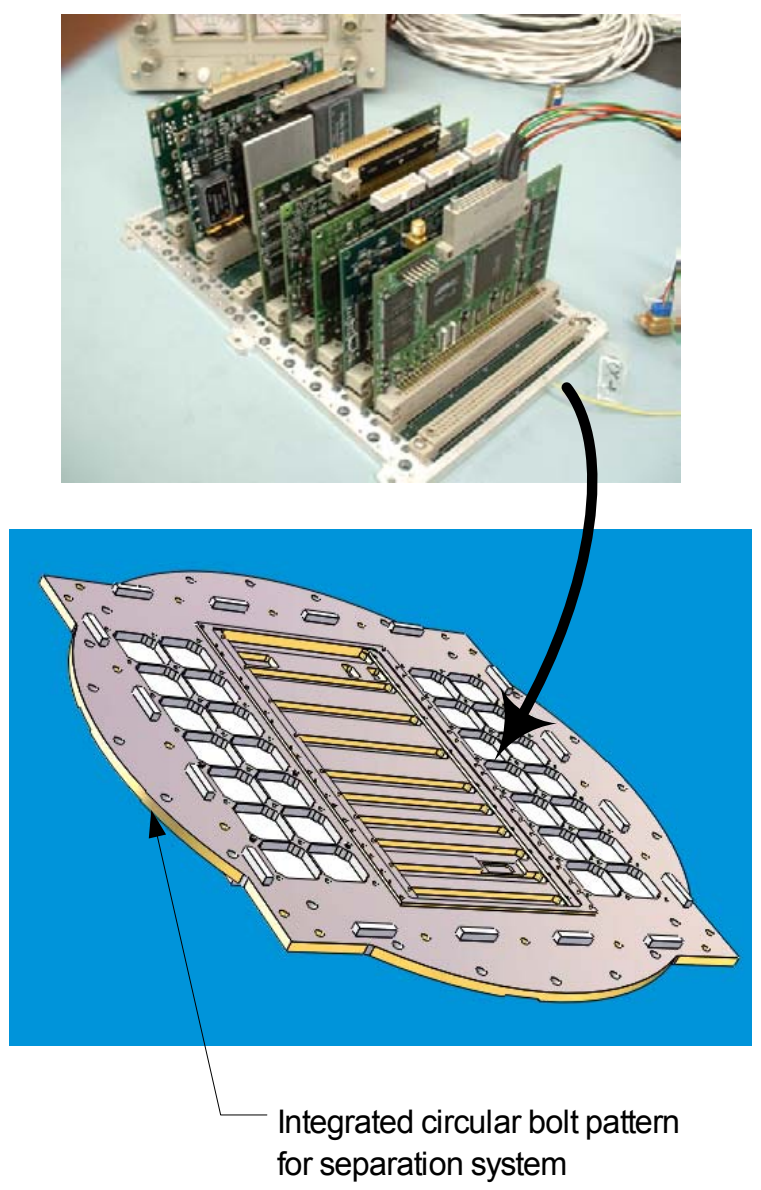

Figure 6-5. The USUsat 2 flight computer integrated in the backplane (top). The aluminum backplane will be directly machined out of the base plate on the flight model (bottom).

The Ortho-Grid zone on each of the panels is surrounded by a 1.5 " to 2 " wide untouched frame $(0.25$ " thick) to add stiffness at the joints, as shown in Figure 6-6. This zone can be used to add hinges and release mechanisms where custom holes must be tapped. The inside zone covered by the OrthoGrid can have openings or windows drilled out to give access through the panel to the outside, for cameras or sensors. The baseline structure can therefore be modified as required for new mission or instrument requirements. This adaptability can accelerate the fabrication process without re-engineering the entire structure. Once the precise modification has been made on the shop drawings, machining can take place. 


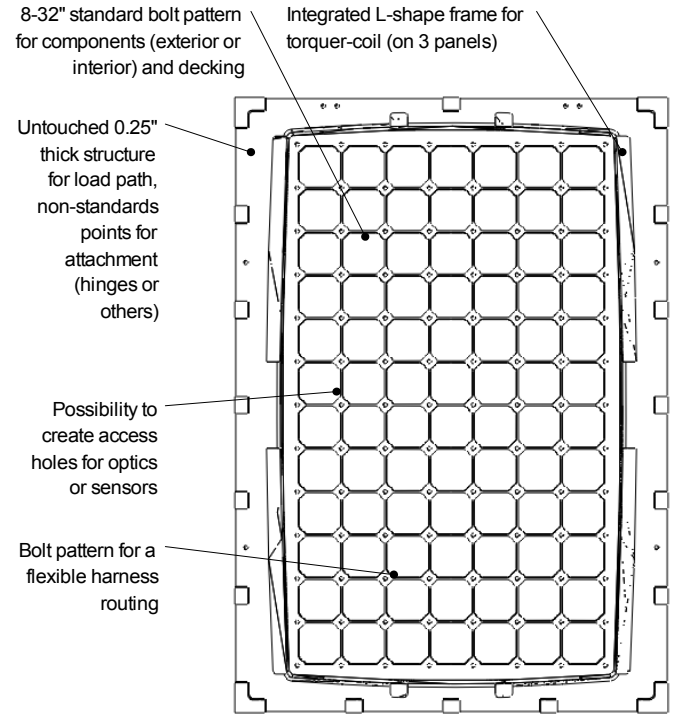

Figure 6-6. USUsat 2 structure panel features for multi-functional and versatile capabilities.

The advantages of the USUsat 2 structure are summarized as follows:

- Standard threaded pattern for versatility in attaching components (inside and outside) and balancing the spacecraft

- Standard threaded pattern for harness attachment (without the use of adhesives)

- Standard threaded pattern for access holes for solar cell wires

- Decking capability by using the same bolt pattern

- Only three different fastener sizes (4-40, 8-32, 10 32 ) to reduce the number of parts and tooling

- Integrated CEE (flight computer) backplane for improved thermal conductivity and to avoid designing a separate part

- Integrated Lightband bolt pattern to avoid designing a separate adaptor plate

- Embedded torquer-coils frame to avoid designing and attaching a separate part

- Vented and self-locking fasteners as default

- Light mass ( 9 kg)

- Contains four points of attachment on each panel for Plexiglas cover protection

- Two side panels contain 0.25 " tapped holes for lifting points when the satellite is entirely assembled

- Made of Aluminum 6061-T6 for high strength and good machinability

\section{$7 \quad$ Decking capability}

The USUsat 2 is designed primarily for a panel-mounted configuration, with all the internal components directly attached to the panels. However, components with high mass or high cantilever configuration will sometimes need to be put on a deck in order to provide good support. In addition, it could be possible for a group of instruments to need to be close together. In these cases they could be assembled on a deck platform. The USUsat 2 design allows the addition decking. Figures 7-1 and 10-2 show decking configurations. The same deck, when empty, can also act as a stiffener. The deck does not contain a skin, or in other words, the OrthoGrid is machined through.

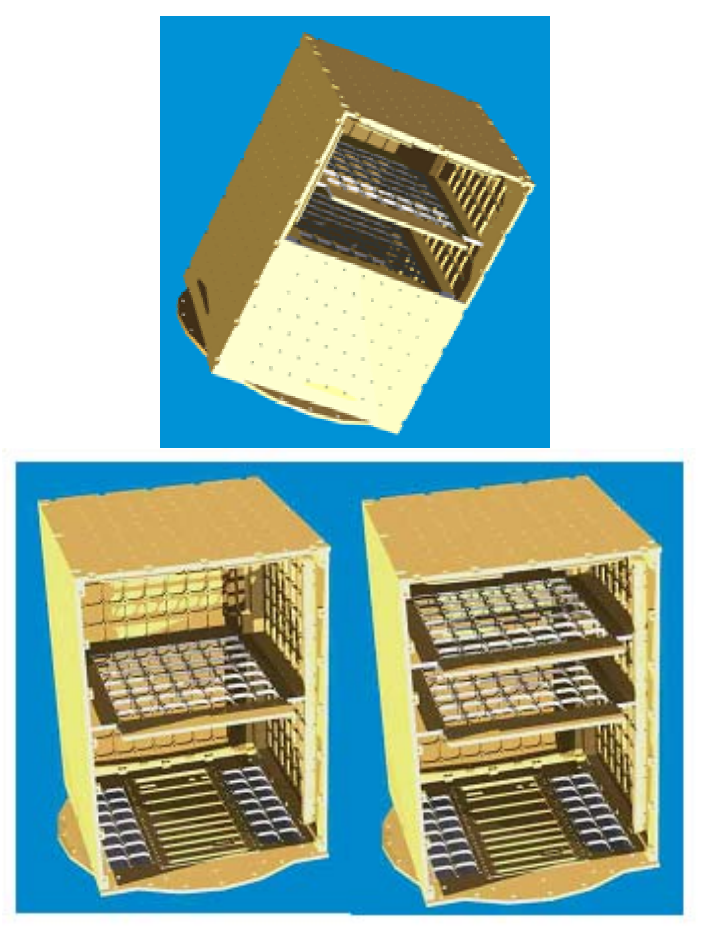

Figure 7-1. Decking capability for one or two decks on the USUsat 2 structure (side panel removed for inside visibility. The second picture shows a half side panel for large external access from decks (sensors, cameras, etc.) 


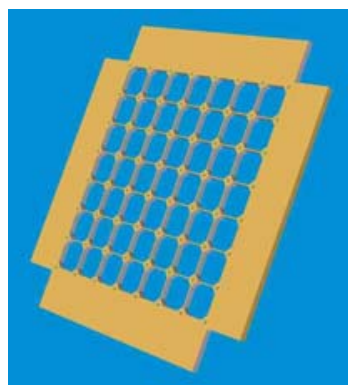

Figure 7-2. Open ortho-grid deck with standard bolt pattern.

\section{Harness}

Through the standard bolt pattern on the structure the harness can easily be attached in a clean configuration when the panels or modules are separated. Pre-harnessing the spacecraft through modules contributes to an open access for tooling and a minimum number of connectors. Once the harness is pre-assembled onto one module, it is attached to one connector that is attached to the bottom panel containing the flight computer. (For communication, coax cables will require another kind of connector).

The bolt pattern allows versatility with various points for rigid attachment of the harness thus avoiding the need to use tape (See Figure 4-2).

Since all modules are pre-wired, an extender harness can connect them to the bottom panel for initial electrical test without requiring a closed, or fully assembled, satellite. This offers an open access for components in the event debugging is required.

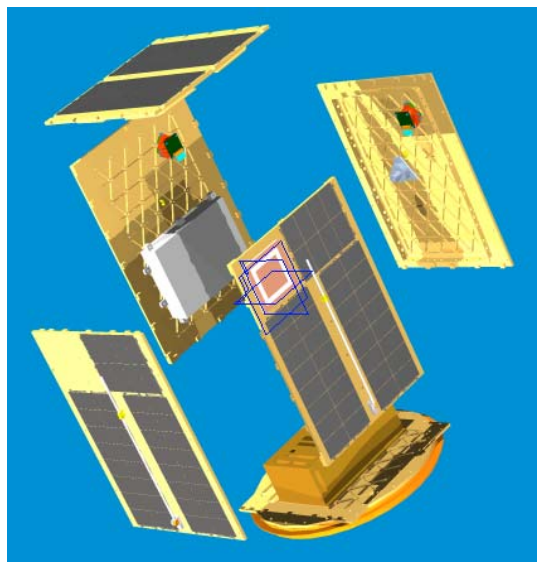

Figure 8-1. Exploded view of USUsat 2 in the basic or "light" configuration as it is for the UNIII mission

\section{Separation system}

The separation system used on the USUsat 2 is attached directly to the bottom panel of the structure by following a circular bolt pattern. The current mechanism utilized is the Lightband provided by Planetary System Corp (see Figure 9-1). If another circular separation system is required, the bottom panel can be readapted according to the standard. On their Structure for Auxiliary Payload (ASAP), Arianespace uses their own mechanism, which can be adapted to the bottom panel of the structure by modifying the bolt pattern. The structure of the USUsat 2 does not have an adapting plate. Here the bolt pattern is directly machined out of the bottom panel thus eliminating an external part and an assembly step.

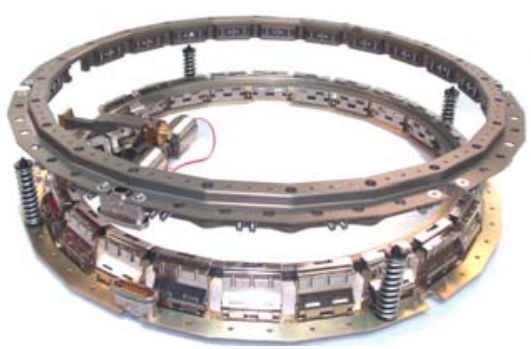

Figure 9-1. The Lightband separation system from Planetary System Corp used on USUsat 2.

\section{Internal light Configuration (panel mounted)}

The USUsat 2 "light" configuration (see Figure 10-1), or basic configuration, shows the simplest possible configuration in terms of packaging and manufacturing. The basic package of the light model is assembled with a panel-mounted configuration. The Common Electronic Enclosure or CEE box sits on the bottom panel where all the harnessing from adjacent panels converges. The solar panels are also body mounted thus eliminating the need to have deployable panels or external structures, such as Honeycomb panels, attached. The only deployable components are four dipole antennas. These can be replaced with patch antennas is requirements allow.

The current USUsat 2 design can still accommodate additional "light" payloads. Margins in power and mass are still available on-board.

This configuration contains the minimum capability required for mission operation with a light 
payload capability. The assembly strategy for all the configurations follows the same principle: preassemble and test the spacecraft through modules and process to the top assembly and final test prior to launch.

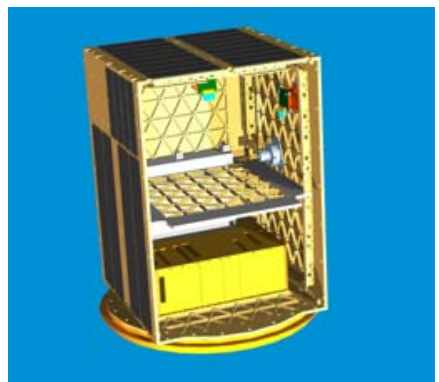

Figure 10-1. Higher stiffness with multiple attachment points on the side.

\section{Internal Heavy Configuration (hybrid-panel mounted + deck mounted)}

As the panel-mounted configuration has its limit in terms of mass and components with a high cantilever beam characteristic, the need to enlarge the surface area perpendicular to the main axis (launch) can be accomplished by adding decking capability. This also offers the advantage of having a family of interconnected components being tested together (vibration and thermal) on their deck. The integrated bolt pattern can quickly accommodate an extra surface through the open OrthoGrid structure. The weight of an additional deck made of aluminum can be up to $\sim 0.850 \mathrm{~kg}$. The panel can be attached between the four side panels. Like the main panels or modules of the spacecraft, the deck can be preassembled with its respective components, preharnessed, tested separately, and then integrated into the rest of the satellite. Figure 11-1 shows the internal configuration with one deck.

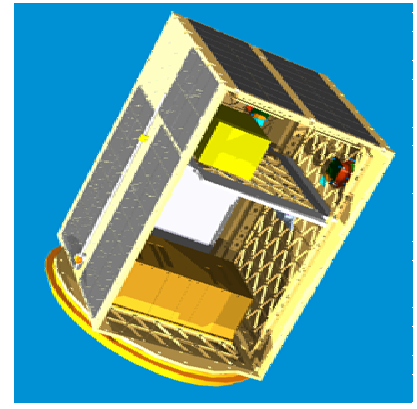

Figure 11-1. USUsat 2 internal configuration (Heavy I) with one deck for component mounting and/or as an additional stiffener. Mass range $=25$ $40 \mathrm{~kg}$.
The benefit of configuring the spacecraft with decks is the additional room available for packaging, particularly with heavy components. In most cases, in this "piggy-back" panel orientation, the principal axis will be aligned with the flight trajectory, reducing laterals loads. Figure 11-2 demonstrates the same function with two decks.

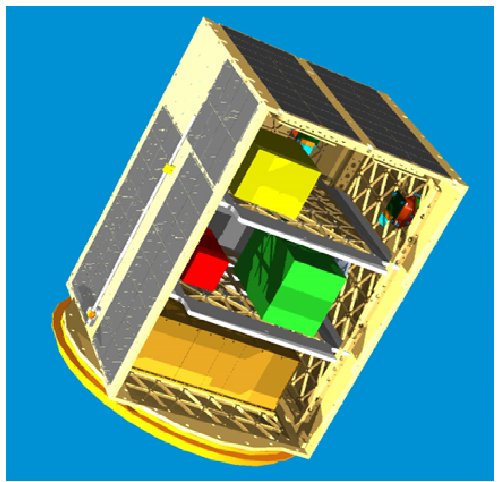

Figure 11-2. USUsat 2 with a dual deck configuration (Heavy II) allowing heavy components to be separately mounted and tested. The deck can avoid heavy components being in a cantilever beam configuration on a panel. Mass range $=30-50 \mathrm{~kg}$.

Another possible configuration is to remove the top panel of the structure, allowing the top deck instruments to be exposed to the exterior. This configuration is often seen with the Surrey Microsatellite-70 bus. It offers direct access or field of view for multiple sensors that need direct exposure to the outside. The top deck also plays a role in closing the primary structure with or without being at the very end of the spacecraft.

\section{USUsat 2 "Light", external light modular configuration capabilities}

In order to increase the capability of various configurations the external envelope of the spacecraft must be versatile as well. The external design is driven by many factors such as solar cells, communication antennas, access holes for inside sensors or cameras, attachment for deployable mechanisms and separation mechanisms and external connectors for testing purposes. The structure must show certain versatility while maintaining the integrity of the spacecraft. The USUsat 2 concept responds to this challenge by approaching the problem in a simple way: a rectangular primary 
structure that can accommodate internal components and external components with the minimum amount of modification from the basic structure model.

The "light" external configuration of USUsat 2 uses the skin of the structure to directly attach light components such as patch antennas or solar cells. This arrangement of panel-mounted solar cells eliminates pre-mounting the cells onto a separate structure that will then be attached to the spacecraft. The traditional method for attaching the solar cells is to assemble them on a separate structure such as a HoneyComb panel and then to attach the HoneyComb to the primary structure of the spacecraft. To reduce the number of parts and assembly operations (applying DFMA), the USUsat 2 combines the two steps- "killing two birds with one stone." The "light" configuration does not integrate "heavy" deployable structures such as solar panels. The principal source of power comes from the independent orientation of the spacecraft with respect to the sun, assuming that the bottom panel is nadir pointing. The outside configuration can also take advantage of the versatility in attachment offered by the bolt pattern.Figure 12-1 shows the micro-satellite for the UNIII configuration with body mounted solar cells.

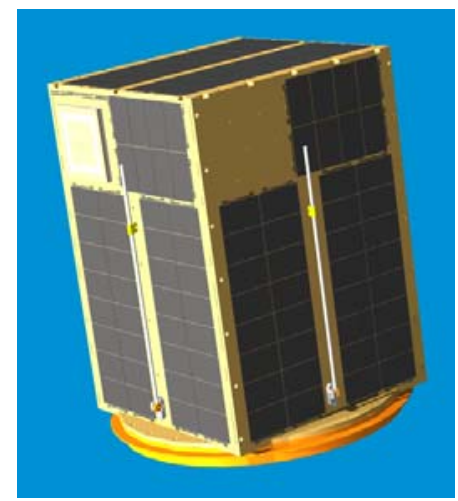

Figure 12-1. External light configuration with panel mounted solar array as designed for the UNIII mission

\section{USUsat 2 "Heavy", with external deployable}

It is often undesirable to include deployable panels, thus reducing risk, avoiding costly components, and eliminating extensive testing; however, the industry offers a wide range of reliable mechanisms for small satellites to control deployable components. Figure 13-1 shows one such component. These mechanisms can be prohibitively costly for a university program. It is possible to spend more than $\$ 100 \mathrm{~K}$ to install four hinges with release mechanisms on a small satellite in addition to the schedule cost of lead-times on the order of months. Still, whenever the average power required on orbit cannot be supported by solar mounted cells alone, the option of adding deployable panels must be considered.

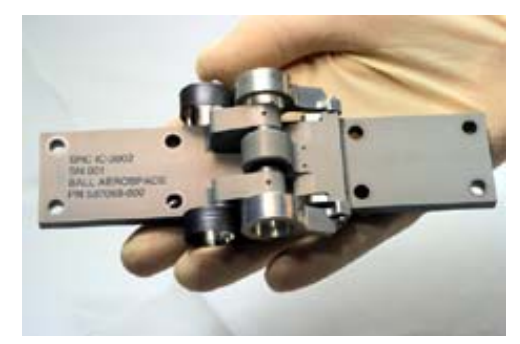

Figure 13-1. The Starsys SH-18010 hinge for deployable panel (mass is $200 \mathrm{~g}$.)

For situations where deployable components are required, small deployable surfaces made of closed aluminum IsoGrid or thin Honeycomb panel can be used, coupled with off-the-shelf hinge and release mechanisms. With this arrangement, solar cells can be added to improve the orbit average power when increased electrical power generation is required. It is also possible to keep the rest of the cells mounted on the other side panels or removed according to needs of the design.

The assembly of the panels is simple. The deployable solar panel is first attached to the side panel with the other components to form a module. Then the module is connected mechanically and electrically to the rest of the spacecraft. The versatility and efficiency of such a configuration can also be increased by providing a fixed angle orientation on the deployable panel that will give the satellite the same geometric aspect as hexagonally shaped structure. The deployable components can first be assembled on their respective panels or modules and pre-tested with in the assembled configuration, thus building confidence prior to spacecraft integration. Figure 13-2 shows the USUsat 2 with a small size deployable solar panel to increase the top surface area. 


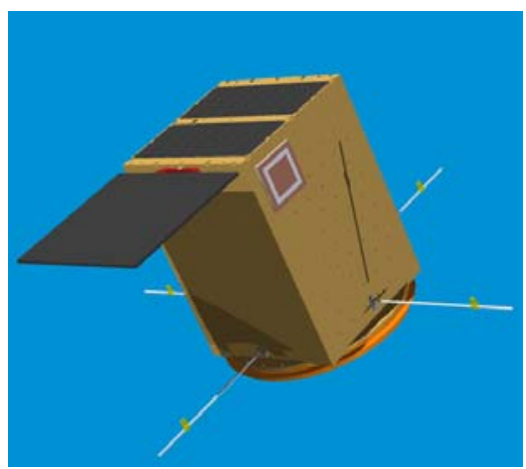

Figure 13-2. One solar panel deployable to increase on-orbit average power assuming. Top panel is facing zenith. Open and closed position

Two small deployable panels can also be added if needed, as shown in Figure 13-3. In this configuration, the location of the attachment points for the hinges and release mechanisms will be identical for all the structures, simplifying the documentation if the same design must be duplicated. If instruments, such as sensors or cameras, require pointing in the zenith direction, to have access to the top panel, it will be necessary to increase the top surface exposure for solar cells with the help of adjacent deployable panels.

Another situation requiring deployable panels would be high orbits, such as a geo-transfer orbit, where the time exposed to the sun is considerable and can have a strong impact on the thermal balance of the spacecraft. Deployable panels have the benefit of not transferring these high heat loads to the principal structure and the instruments.

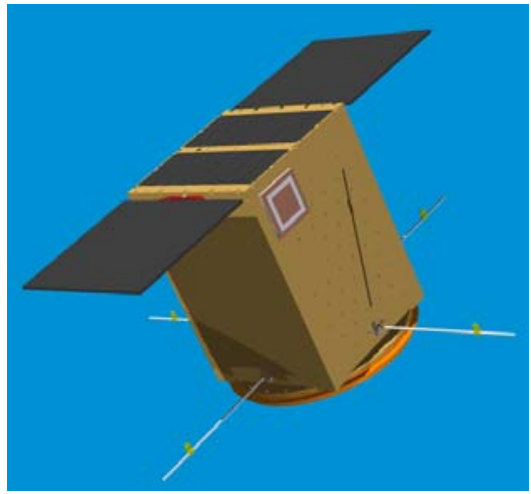

Figure 13-3. USUsat 2 with two small-deployed panels.

The two-panel configuration can also be increased to four solar panels. Here the driven requirements are the selected orbit, the average power required, and attitude. Figure 13-4 shows the USUsat 2 satellite with four deployed panels.

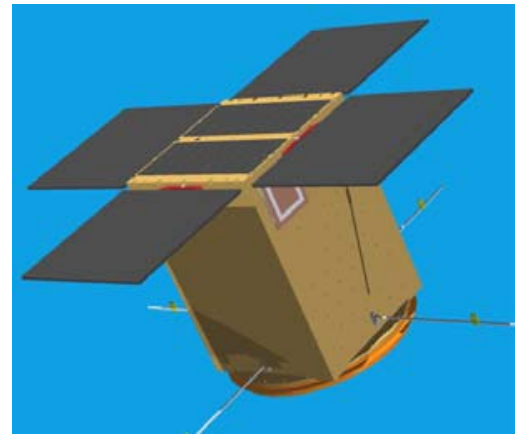

Figure 13-4. USUsat 2 with four solar panels. The top panel can have the cells removed and accommodate instruments or cameras if needed.

Figure 13-5 shows the USUsat 2 solar panels deployed with a fixed angle, other than $90^{\circ}$, for better solar power generation over the sun exposure. This configuration gives to the spacecraft a solar exposure to the cells similar to a hexagonal shaped structure with body-mounted panel such as for USUsat I/ION-f. With a modular design, the satellite offers many possible configurations to make the best adaptation for a required mission.

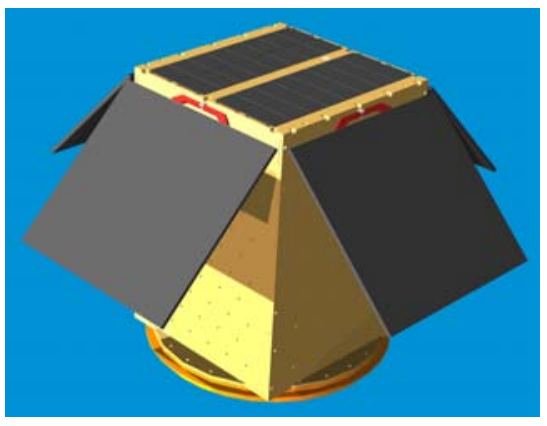

Figure 13-5. The geometry can act as hexagon shaped structure by giving a fixed deployed angle to the solar panels that would have body-mounted cells.

The deployable panel additions described above are hinged relative to the top surface. If a mission requires one of the side panels to become the nadir orientation for instrument purposes, then it is possible to deploy the panels from the large sides of the structure. Figure 13-6 shows such a configuration, with a deployable as large as the side panels. This 
size of deployable may not be necessary for most missions, but does allow a larger number of solar cell strings. This deployable panel, like those previously described, can be made of light thin honeycomb, and actuated by an off-the-shelf hinge mechanism. It is important to note that the release mechanism does not necessarily have to be located on the inside of the spacecraft. If space is a constraint when using panelmounted configurations, it is possible to locate the release mechanism on the outside and connect it to the inside electronics.

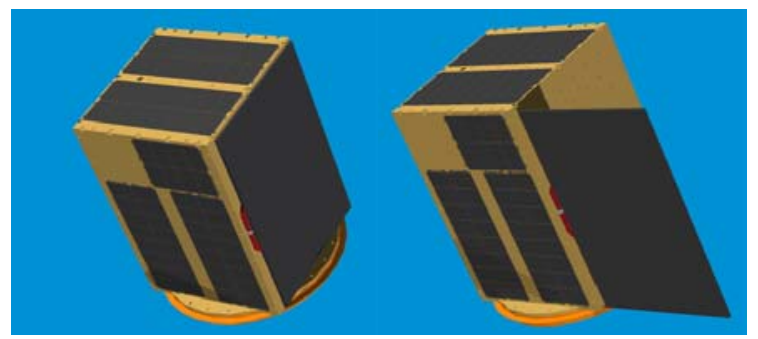

Figure 13-6. USUsat 2 with large deployable panel.

Figure 13-7 shows the USUsat 2 with the greatest amount of deployable area in a single plane. A combination of a top deployable panel coupled with two large side panels can be used if a large quantity of power is required. The panel oposite the visible face can accomodate holes in the structure and be pointed nadir if instruments require. These instrument could also be either directly located on the panel, or on a deck.

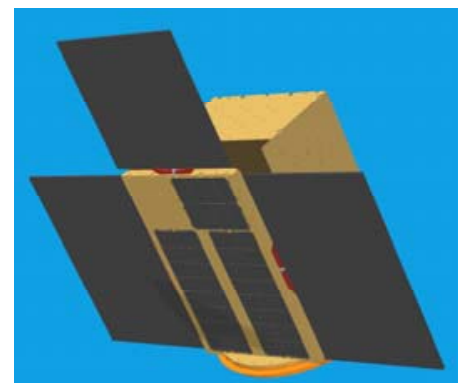

Figure 13-7. USUsat 2 with a configuration capable of providing a high surface area for solar power generation.

\section{Case model}

The following represents a case where the USUsat 2 platform could be applicable. A microsatellite is needed to carry measurement sensors. The instruments need to be space proven and flight-tested prior to a larger scale mission with a more important financial involvement. The instrument requirements indicate that to perform the mission they must be oriented toward the earth through the majority of the mission timeline. High power consumption is assumed. The customers are seeking a micro-satellite platform that could carry the instruments as payloads and provide the entire necessary interface to operate the mission and download the data to earth. The customers want to verify the technology as quickly as possible. The instruments are assumed to be in the design process or already designed. The instrument qualification process should already be carried out by the manufacturers. The remaining tasks to perform are software design and electronic hardware that will communicate to the payload, the electrical interface to provide power, and the data interface to download the information. A mechanical support interface must also be provided for thermal and structural integrity.

If, at this point, the USUsat 2 platform with its bus has flight heritage, the USUsat 2 baseline design is qualified and all technical information related to the design is ready for production. The structure and all the sub-systems are can be reused in this case. Of course, there will be need to do more system engineering at the mission level and more analysis at the sub-systems level; however, it is assumed that the structure remains the same with the exception that the top panel must be removed and a deck must be included near the top to accommodate the instrument in a protected zone (perhaps to protect against tooling accidents). The torquer coil frame carried by the top panel can now be integrated and machined in deck. The communication system is remains from the original configuration, as well as the C\&DH subsystem. It is possible that additional buffer memory will be needed, depending on the ground station opportunity, orbit, and altitude. The extra memory could be located on the bottom panel near the C\&DH box. Since the payload is new to the USUsat 2 design team, software work must be completed in to command and control the instruments. The power sub-system is also carried over from USUsat 2, with only an increase in solar cell area created by the addition of four deployable panels oriented with a 45 degrees angle toward nadir. The four sides of module 1,2,3 and 4 are also covered with cells, as in the original configuration. The battery box is also kept as-is. With the design evolution from the USUsat 2 platform, analysis such as thermal and structural can be completed using modified versions of the original models. For the structure analysis, loads from the new instruments must be evaluated as well as the presence of deployable panels. Since it is possible that the 
mission will be launched from a different launch vehicle, the stiffness requirements would require evaluation using the new loads. The ADCS is also reused, providing full 3-axis control of the spacecraft. Figure 14-1 illustrates the new spacecraft configuration. The design of this spacecraft would be rapid, due to the use of components common to the USUsat 2 satellite.

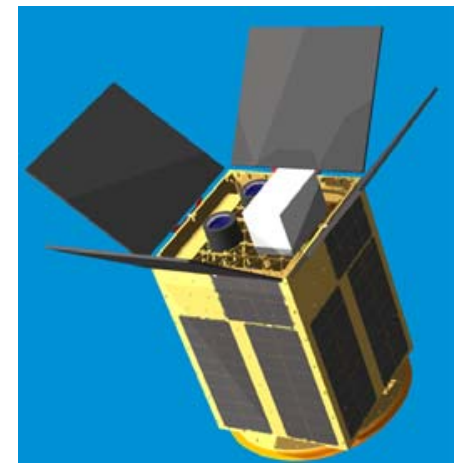

Figure 14-1. Possible new configuration for the application of USUsat 2 in the described example. The regular top panel has been removed and a deck has been positioned upward to provide support on the structure and an instrument platform.

\section{Testing through modules}

The USUsat II testing approach is to treat pieces of the satellite as pre-assembled modules that will be considered as sub-structures. For example, if a camera assembly needs to be tested on a vibration table, then why not attach to the same fixture all the cameras that will be used in the satellite. The scale of such components for a micro-satellite is small and light (in the order of 100 grams) and therefore can be combined during the test. In this manner, instead of designing one test fixture for one camera, one test fixture is designed that will adapt to the four cameras grouped together. The cost of using the vibration table can be significant; therefore, the cost savings can be significant, when testing multiple components at once. The size of components in a micro-satellite are designed to be compact and light, so using the same table at the same time is also feasible. If this approach is extended to the modules than can be tested separately, then the same conclusion can be made. Rather than having a fixture to adapt to a series of several components, the author proposes that one of the panels of the satellite be assembled with its adjacent components to be a module and become the supporting fixture at the same time. In the case of the vibration tests (and even for the thermal tests) the goal is to design a fixture that will match and hold each module of the satellite, thus saving time and cost in comparison to each module using a separate fixture design. The same fixture can later be used when the spacecraft is integrated, since it will match the bottom panel. The entire process can then become leaner. If a problem occurs at that level, then disassembling the modules is not a difficult task. An open access area to each component on the modules can allow the test engineer to perform functional tests and visual inspections. This is not taking shortcuts in testing but rather taking an efficient and economic approach by capitalizing on the merits of microsatellite architecture. In large satellites, components are usually tested at the sub-system or component level, often with each sub-system as large as a fully assembled micro-satellite.

Another advantage of module testing is that the test environment is closer to the final environment during launch where components are mounted on their respective panel. In other words, testing a panel with all of its components can more accurately duplicate the true launch environment. This will provide more confidence to the team than an "ideal" environment where other components' contribution to induced frequencies is neglected. This testing approach also has the benefit allowing a module to start its test sequence without waiting for other modules to be finalized.

\section{Current status}

The USUsat 2 structure is scheduled to be fabricated in the fall of 2004 at SDL. A 3D laser print model, for use in fit-check and display, is nearing completion. The USUsat 2 Micro-satellite is intended to be fully fabricated in 2005 .

\section{References}

Quincieu J., USUsat 2 Design for Flight and Modular Platform, A Guideline for Mechanical Design, Integration \& Test, Manufacturing and Modular Platform in USUsat 2 Microsatellite. Master Report, Utah State University, Logan, UT, 2004. 